\title{
BMJ Open Impact of using spirometry on clinical decision making and quality of life in children: protocol for a single centre randomised controlled trial
}

\author{
Wicharn Boonjindasup (D) ,1,2,3 Julie M Marchant (D) ,3,4 Margaret S McElrea, ${ }^{3,4}$ \\ Stephanie T Yerkovich, ${ }^{1,3}$ Ian Brent Masters, ${ }^{3,4}$ Anne B Chang ${ }^{1,3,4}$
}

To cite: Boonjindasup W, Marchant JM, McElrea MS, et al. Impact of using spirometry on clinical decision making and quality of life in children: protocol for a single centre randomised controlled trial. BMJ Open 2021;11:e050974. doi:10.1136/ bmjopen-2021-050974

- Prepublication history for this paper is available online. To view these files, please visit the journal online (http://dx.doi. org/10.1136/bmjopen-2021050974).

Received 05 March 2021 Accepted 05 September 2021

Check for updates

(C) Author(s) (or their employer(s)) 2021. Re-use permitted under CC BY-NC. No commercial re-use. See rights and permissions. Published by BMJ.

${ }^{1}$ Child Health Division, Menzies School of Health Research, Charles Darwin University, Darwin, Northern Territory, Australia

${ }^{2}$ Pediatrics, Faculty of Medicine, Chulalongkorn University,

Bangkok, Thailand

${ }^{3}$ Australian Centre for Health

Services Innovation, Queensland University of Technology, Brisbane, Queensland, Australia ${ }^{4}$ Respiratory and Sleep Medicine, Queensland Children's Hospital, South Brisbane, Queensland, Australia

Correspondence to Dr Wicharn Boonjindasup; elm.boonjindasup@menzies. edu.au

\section{ABSTRACT}

Introduction Although spirometry has been available for decades, it is underused in paediatric practice, other than in specialist clinics. This is unsurprising as there is limited evidence on the benefit of routine spirometry in improving clinical decision making and/or outcomes for children. We hypothesised that using spirometry for children being evaluated for respiratory diseases impacts on clinical decision making and/or improves patient-related outcome measures (PROMs) and/or quality of life (QoL), compared with not using spirometry.

Methods and analysis We are undertaking a randomised controlled trial (commenced in March 2020) that will include 106 children (aged 4-18 years) recruited from respiratory clinics at Queensland Children's Hospital, Australia. Inclusion criteria are able to perform reliable spirometry and a parent/guardian who can complete questionnaire(s). Children (1:1 allocation) are randomised to clinical medical review with spirometry (intervention group) or without spirometry (control group) within strata of consultation status (new/review), and cough condition (present/absent). The primary outcome is change in clinical decision making. The secondary outcomes are change in PROM scores, opinions regarding spirometry and degree of diagnosis certainty. Intergroup differences of these outcomes will be determined by $\chi^{2}$ test or unpaired t-test (or Mann-Whitney if not normally distributed). Change in outcomes within the control group after review of spirometry will also be assessed by McNemar's test or paired t-test/Wilcoxon signed-rank test.

Ethics and dissemination The Human Research Ethics Committee of the Queensland Children's Hospital approved the study. The trial results will be disseminated through conference presentations, teaching avenues and publications.

Trial registration number ACTRN12619001686190; Pre-results.

\section{INTRODUCTION}

Of the many possible lung function tests used in clinical care, spirometry is the most widely available, established and used. ${ }^{1}$ As such, many respiratory societies worldwide, for example, American Thoracic Society (ATS) and European Respiratory Society (ERS)
Strengths and limitations of this study

- This randomised controlled trial will provide important information on whether the routine use of spirometry in children being evaluated for respiratory problems impacts a doctor's clinical decision making (compared with clinical review alone) and will thus provide the first high-level evidence that may lead to a change in routine clinical practice.

- Patient-reported outcome measures (anxiety level, quality of life score and opinion towards spirometry) will be undertaken to determine the utility of routine spirometry.

- Although this study is randomised with a control group and concealed allocation, the intervention could not be blinded to the doctors and participants. Therefore, the outcomes are subject to bias as perceptions may influence doctors' management and participants' scoring of the questionnaire(s).

support and/or provide training tools for spirometry testing. Undertaking spirometry is relatively simple as spirometers are portable and relatively inexpensive.

Data from spirometry provides invaluable contribution to the clinical assessment, including assisting in characterising respiratory pathophysiology, grading the severity of lung disorders and monitoring the course of lung disorders and therapeutic interventions. $^{23}$ Also, spirometry adds an objective element which is beneficial in both clinical practice and research. Hence, its use is recommended in many paediatric clinical guidelines including chronic cough, recurrent wheezing, cystic fibrosis and asthma. ${ }^{4-7}$ Other conditions in which spirometry aids in management of children are transfusiondependent disorders, oncology conditions, connective tissue disorders, neuromuscular weakness, chest wall deformities and scoliosis. $^{28}$ 
Data obtained from spirometry differentiate normal lung function from abnormalities affecting airflow (forced expiratory volume in $1 \mathrm{~s}$ ) and lung size (forced vital capacity). It can also provide data on intrathoracic and extrathoracic obstruction when the inspiratory and expiratory loops are evaluated. Generally, spirometry can be reliably performed in most children aged $>6$ years. Improvements in equipment, technology, age-appropriate incentives in spirometer software and modified acceptability and reproducibility criteria for preschool children have meant even younger children (3 years and above) may be able to perform spirometry satisfactorily under the coaching of a well-trained technician. ${ }^{1910}$

Intuitively, spirometry should assist clinicians in assessing and managing respiratory conditions and result in improved patient-related outcome measures (PROMs), for example, quality of life (QoL) of the patients, however, there is limited published evidence. In the current era of evidence-based medicine, the effect of spirometry on clinical outcomes has rarely been studied with the few paediatric studies published looking only at its use in asthma management. Nair et $a l^{11}$ found that spirometry changed management in $15 \%$ of children with asthma. When spirometry did change treatment decisions, they were more likely to increase $(75 \%)$ than maintain $(20 \%)$ or decrease $(5 \%)$ therapy. Holt $e t a l^{2}$ found that $30 \%$ of paediatric asthma exacerbation treatment plans were changed after clinicians viewed spirometry, with an increased percentage of patients receiving steroid, bronchodilator or yellow zone treatment. Finally, Abramson et $a l^{13}$ undertook a two clustered randomised controlled trials (RCTs) of spirometry integrated into regular general practice-based medical review for children with asthma over the duration of 1 year. They found that neither RCT demonstrated a significant improvement in health-related QoL with the use of spirometry compared with not using (adjusted difference of Paediatric Asthma Impact Scale -0.2 (95\% CI -4.9 to 4.6 ) for the first trial and of Paediatric Asthma Quality of Life Questionnaire 0.17 (95\% CI -0.15 to 0.5 ) for the second) nor a change in written asthma action plan (OR 1.11, 95\% CI 0.43 to 2.87).

While spirometry is widely advocated, it is currently underused. Dombkowski et $a l^{14}$ reported that only half of surveyed family physicians and general paediatricians use it in children and adults with asthma, and only $21 \%$ routinely use spirometry in asthma guidelinerecommended situations, that is, establishing an asthma diagnosis, classifying asthma severity and classifying asthma control. ${ }^{14}$ Another study by Blain and Craig ${ }^{15}$ reported that only $10 \%$ of paediatricians used spirometry consistently on each asthma visit. Further, Bianchi et $a l^{16}$ found that only one-third of children with asthma were referred for spirometry and only one-half of hospitalised children with asthma underwent spirometry during 12-month follow-up. The low utilisation of spirometry on a day-to-day basis outside of respiratory-focused practices is not surprising due to the limited published data supporting its benefits.

Given the paucity of relevant data, we are undertaking this RCT to compare clinical outcomes of outpatient consultation 'with spirometry' versus 'without spirometry' to assess the benefit of having spirometry data for both clinicians and patients.

\section{Study objectives and hypotheses}

Our primary question is: Does the routine use of spirometry improve the clinical decisions/management of children with suspected or known lung disease? We hypothesise that the routine use of spirometry in children managed by respiratory paediatricians in outpatient clinics alters clinical decision making in diagnosis and/or management.

Our secondary aims are to: (1) determine whether the routine use of spirometry in children impacts on diagnostic certainty and PROMs and (2) quantify the benefits of routinely using spirometry in clinical practice assessed by a 10 -point Likert scale. ${ }^{17}$ Our secondary hypothesis is that the integration of spirometry into outpatient consultations with respiratory paediatricians improves diagnosis certainty, PROM(s), specifically in emotional and social domains, evaluated by State-Trait Anxiety Inventory $(\mathrm{STAI})^{18} 19$ and/or Parent-Proxy QoL questionnaire for paediatric chronic cough (PC-QoL) ${ }^{20}$ for those with chronic cough.

\section{METHODS AND ANALYSIS \\ Study setting and design}

We are conducting a single centre RCT with concealed allocation involving children seen at the Department of Respiratory and Sleep Medicine at the Queensland Children's Hospital, Brisbane, Australia. Our study design is summarised in figure 1 , and is in accordance with the recommendations of the Standard Protocol Items: Recommendations for Interventional Trials guidelines. ${ }^{22}$ We recruited our first participant on 17 March 2020 and our study is ongoing with the study anticipated to be completed by early 2022 .

\section{Study population}

Our inclusion criteria are: (1) children with parent/ guardian in attendance and able to provide written consent, (2) children aged 4-18 years able to perform reliable spirometry, (3) parents/guardian able to complete the study questionnaire(s) and (4) children whose respiratory physician is willing to participate in the study.

Exclusion criteria are: (1) previously enrolled or (2) contraindication for spirometry including presence of acute dyspnoea, pneumothorax, haemoptysis, vital signs instability, lung cyst or bleb and recent ( $<3$ weeks) thoracic or ophthalmic surgery. 


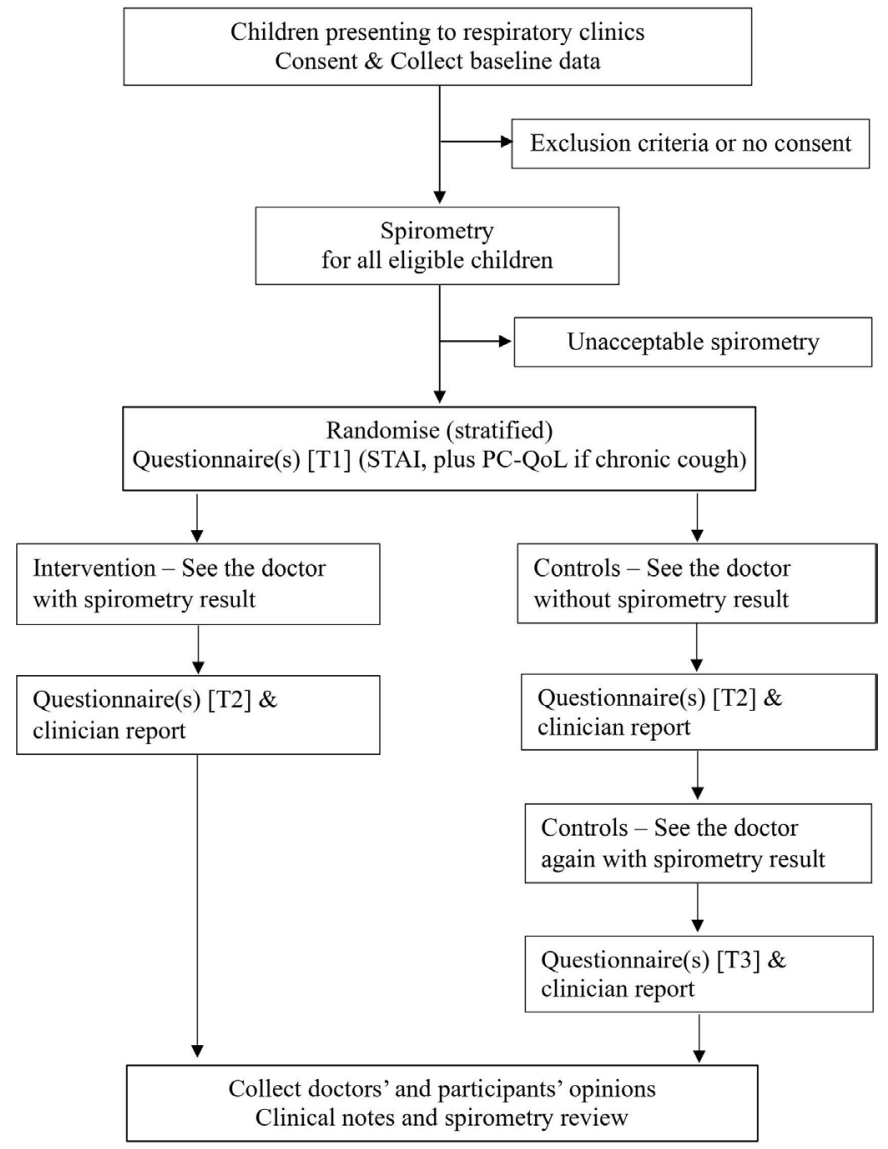

Figure 1 Schematic study design. PC-QoL, Parent-Proxy QoL questionnaire for paediatric chronic cough; STAI, StateTrait Anxiety Inventory.

\section{Recruitment}

Parents/guardians with potentially eligible children are approached during a scheduled respiratory clinic appointment. A team member (WB) discusses the study using the parent/guardian information sheet. If the parent/guardian gives written informed consent and the child is able to perform acceptable spirometry then the child enters the study and is randomised.

All families approached are recorded in a screening $\log$ with details including name, date, whether informed consent was obtained or reason for refusal, whether the child was eligible and randomisation number if enrolled in the study.

\section{Randomisation and allocation}

A computer-generated permuted block (sizes of 2-6) randomisation sequence, generated by a statistician external to the study team, was prepared prior to commencement of the study. The randomisation is stratified by type of consultation (new patient or review), and presence of chronic cough condition (present or absent). On enrolment, the child is assigned to the next number on the stratified list that is opaque, that is, the group allocation is concealed from investigators until the participant is recruited. Children are randomised to either routine use of spirometry (intervention) or delayed use of spirometry (controls) (figure 1). Due to the obviously noticeable difference in intervention versus control groups, blinding is not possible.

\section{Intervention groups}

For both groups, the clinical management is at the discretion of the treating specialist. At baseline (T1), all participants undertake baseline PROM surveys $\left(\mathrm{STAI}^{18}{ }^{19}\right.$ and if cough is present, the PC-QoL ${ }^{2021}$ ) and are randomised to one of the two groups: intervention group where the doctor undertakes the consultation with the spirometry results being available, or controls where the doctor undertakes the initial consultation without the spirometry results.

After the consultation, the parents in both groups complete the questionnaire(s) for the second time (T2). At this same time point, the doctor is also asked to fill a data collection sheet regarding the child's diagnosis and management. This completes the study for the intervention group.

In the control group, the doctor is then presented with the child's spirometry and completes the consultation. After completion of the consultation, the parents complete the same questionnaire(s) for a third time (T3) and the doctors complete a final data collection sheet with any change in diagnosis or management after spirometry results are known. Despite the delayed time point of presenting the spirometry to respiratory paediatricians, by the time the child leaves the clinic, all patients would have received the same standard of care. No restrictions on concomitant care were applicable in this study.

\section{Data collection}

An outline of study procedures (all occurring during a single outpatient visit) is summarised in table 1. Data are collected from the interview of the parent/guardian and electronic medical records. On enrolment, demographic data, medical history, medications, tobacco smoke exposure and anthropometrics are recorded. Physical examination is performed and recorded by the treating doctor.

All children are tested using Vyntus PNEUMO or Vyntus SPIRO spirometers operated through SentrySuite software (Carefusion Germany $234 \mathrm{GmbH}$, Hoechberg, Germany) undertaken by experienced paediatric respiratory scientists in accordance with standard guidelines (ATS and ERS criteria for lung function testing). ${ }^{123}$ The measured values are compared with predicted reference values from the Global Lung Function Initiative ${ }^{24}$ based on the patient's height, age, race and sex.

As mentioned above, depending on study group, the parents complete the same questionnaire(s) two or three times. These time points are prior to visiting the doctor (T1), after visiting the doctor (T2) and after second consultation with the doctor for controls only (T3). The questionnaires are: (i) STAI for all patients and (ii) PC-QoL for patients with cough. For both questionnaires, parents are given a hard copy of questionnaire(s) to 
Table 1 Timeline of study procedures during a single outpatient visit

\begin{tabular}{|c|c|c|c|c|}
\hline & $\begin{array}{l}\text { Before } \\
\text { seeing } \\
\text { doctor }\end{array}$ & $\begin{array}{l}\text { After seeing doctor } \\
\text { without spirometry } \\
\text { (only controls) }\end{array}$ & $\begin{array}{l}\text { After seeing } \\
\text { doctor with } \\
\text { spirometry }\end{array}$ & $\begin{array}{l}\text { Independent } \\
\text { review }\end{array}$ \\
\hline Written informed consent & $\sqrt{ }$ & & & \\
\hline Randomisation & $\sqrt{ }$ & & & \\
\hline Medical history interview and chart review & $\sqrt{ }$ & & & \\
\hline Spirometry with bronchodilator testing & $\sqrt{ }$ & & & $\sqrt{ }$ \\
\hline $\mathrm{STAl} \pm \mathrm{PCQ}$ oL assessment & $\sqrt{ }$ & $\sqrt{ }$ & $\sqrt{ }$ & \\
\hline Opinion survey & & & $\sqrt{ }$ & \\
\hline
\end{tabular}

PC-QoL, Parent-Proxy QoL questionnaire for paediatric chronic cough; STAI, State-Trait Anxiety Inventory.

complete on a self-report basis, with the investigator available if any clarification is needed.

The STAI is a long-standing psychological inventory frequently used to measure anxiety. It consists of two 20-item scales measuring 'state' or anxiety about an event, and 'trait' or anxiety proneness as a personality trait. In responding to the STAI, subjects rate their intensity of feeling for each item on a 4-point Likert scale. Based on its scoring key, the score of each of the two scales (state or trait) ranges from 20 to 80 , where higher scores reflect higher levels of anxiety. ${ }^{18} 19$ A license to reproduce/ administer STAI was purchased from Mind Garden on 15 March 2020.

The PC-QoL is a validated parent-proxy quality of life measure specific for children with chronic cough. ${ }^{20}$ The 27-item PC-QoL addresses parents' perception of three domains: the psychological (11 items), physical (11 items) and social (5 items) effect of their child's cough. Subjects are required to use a 7-point Likert-type scale to rate their perception (level of worry/frequency of negative feelings); the highest intensity of perception gives a score of 1 and absence of perception gives a score of 7 . Hence, higher scores reflect better QoL. For interpreting health-related QoL changes, the minimally important difference for the PC-QoL is $0.9 .^{25}$

Prior to leaving the clinic at the completion of the consultation, the parents also score on a 10-point Likert scale 'how much did the spirometry help with this clinic visit?', while the doctors are asked to score three aspects of its use; 'how much did the spirometry (i) contribute to general management, (ii) increases confidence in clinical practice and (iii) aid education/counselling with each patient?' on a 10-point Likert scale. The unipolar scale of 1 to 10 is anchored by increasing degree of agreement, which 1 means 'not at all', 5 means 'somewhat' and 10 means 'very much so'.

Each participant completes the study on the day of enrolment when all study related forms have been completed and outcome data collected. All data are documented on paper-based case report forms (CRFs) using standardised data collection sheets.

\section{Exit criteria during the study}

Exit criteria are defined as occurrence within the visit of any of the following: (1) spirometry is unacceptable on independent review, (2) the doctor accidentally viewed the spirometry at the start of consultation in the control group or (3) parents withdraw consent for participation, are unable to comply with study intervention or if spirometry-related serious adverse event occurred.

\section{Outcome measures}

Our primary outcome is the proportion of children with any change in clinical decision making (diagnosis and management) and change scores between groups at T2 (figure 1). This consists of an a-priori list that consists of (i) any change in diagnosis based on two categories (disease and severity) and (ii) management based on four categories (medication, investigation, follow-up schedule and education). Change in each category is dichotomised as 'yes' or 'no'. Each 'yes' scores one point, so the range in change score is 0 to a maximum of 6 ( 2 points from change in diagnosis and 4 points from change in management).

Our secondary outcomes are:

1. Change of the PROM scores (STAI $\pm \mathrm{PCQOL}$ ) assessed at $\mathrm{T} 2$ compared with $\mathrm{T} 1$ between two groups.

2. Opinions relating to the benefit of integrating spirometry into clinical practice are included as secondary outcomes. A 10-point Likert scale with a series of statements each designed to view a construct from a slightly different perspective is leveraged.

3. Degree of diagnosis certainty (definite, probable or doubtful) assessed at T2, between both groups.

4. In the control group only, changes in the primary outcome and secondary outcomes 1 and 3, (T3 vs T2).

\section{Sample size}

The sample size is based on our primary outcome, the proportion of children with any change in clinical decision making. We wish to detect a significant difference between the intervention and control groups. We assume the proportion in the population is $30 \%$ (Ho: $\mathrm{p}=0.30$ ). To 
find $45 \%$ proportion of children with any change (alternative $\mathrm{p}=0.45$ ) with $5 \%$ significance (alpha $=0.05$, two-sided) and $90 \%$ power (power $=0.90$ ), we require outcome data from 105 children (rounded up to 106 children). We did not account for any dropouts as this is a single visit study where a dropout rarely occurs. Participants who withdraw will be replaced to reach the total children of 106 .

\section{Data management and statistical analyses and reporting}

CRFs are kept confidential and locked. Access to the data is available to research team, unless required by legislative or regulatory agencies and the Human Research Ethics Committee (HREC). No identifying information of the enrolled participants will be included in study reports in order to protect confidentiality.

Data coding and entry will be conducted in accordance with good clinical practice. Data is being entered directly into an SPSS (version 26) database. Intention-totreat analyses regardless of subsequent management will be used. We plan to develop a complete statistical analysis plan prior to data analyses, as done for our previous major RCTs. ${ }^{26} 27$

For our primary aim, the impact of using spirometry on change in diagnosis and management will be determined by the proportion of children with any change and change scores at the T2 time point. Between the two groups, the proportion will be compared using $\chi^{2}$ test to determine the OR with $95 \%$ CIs. The difference of change scores will be examined by t-test or Mann-Whitney $\mathrm{U}$ test depending on normality of the data.

For secondary aims:

1. Change in PROM (STAI $\pm \mathrm{PCQOL})$ score from T1: The difference of the change between groups assessed at T2 will be compared using t-test or Mann-Whitney test.

2. Opinions towards spirometry quantified by 10-point Likert scales: The result will be reported as mean with SD or median with 25 th-75th percentile.

3. Degree of diagnosis certainty as definite, probable and doubtful for both groups assessed at T2 will be reported as frequency.

4. For controls only, outcomes at T2 and T3 will be compared. These outcomes include change in diagnosis and management, change scores of clinical decisions, change of PROMs and degree of diagnosis certainty. Difference of the outcomes between T2 and T3 will be analysed using McNemar's test, paired t-test/Wilcoxon signed-rank regarding data characteristics.

\section{Patient and public involvement}

Patients and public were not involved in the initial study design but were consulted subsequently.

\section{Ethics, dissemination and safety monitoring}

Ethical clearance was granted by the HREC of the Queensland Children's Hospital (HREC/19/ QCHQ/58722; protocol V.1.3 dated 1 September 2020). We will publish the results in a major medical journal (using the International Committee of Medical Journal
Editors author guidelines) and share the outcomes with the academic and medical community, funding and relevant patient organisations. Professional writers will not be used.

During the study, participants may report any solicited and spontaneous adverse events at any time. All adverse events are being monitored and serious or unexpected adverse events will be reported to the HREC.

\section{DISCUSSION}

We are currently undertaking a single centre openlabel RCT to address the question of whether spirometry integrated into outpatient care, compared with not using spirometry, impacts the clinical decision making of specialist respiratory paediatricians and PROMs. The outcomes and time points were chosen carefully as described below.

\section{Rationale for our chosen outcome measures and time frame}

To measure the influence of using spirometry on clinical practice, choosing valid outcomes informed by consumers. From the doctor's perspective, spirometry should contribute positively to clinical practice for it to be standard practice. Published observational studies also show that $15 \%-30 \%$ of asthma management changed when spirometry was added to the practice. ${ }^{11} 12$ Considering that spirometry plays a plausible role in decisionmaking to diagnose and/or treat patients with suspected or known respiratory conditions, these outcomes were chosen when developing our study design. Further, we clarified the outcomes as two categories of diagnosis: disease and severity, and four categories of management: medication, investigation, follow-up schedule and education. Therefore, the impact of spirometry can be clearly identified.

From the patient's viewpoint, we aim to determine the effect of our intervention (ie, use of spirometry) on PROMs, especially in emotional and social domains, when patients attended the doctor consultation. PROMs are now considered essential for high quality clinical research in order to reliably measure and evaluate the efficacy of an intervention. As young children are unable to adequately communicate their opinion, the standard of PROMs assessment is to approach parents as proxy assessors. In addition, illness of the child usually puts a strain on the whole family, especially the parent or carer. The parent's own opinions and QoL are undeniably relevant as an indirect measure of the child's QoL. Thus, PROMs used in paediatrics concern parents or carers themselves.

In this study, both the STAI and PC-QoL are employed for assessing PROMs to maximise relevant data without overburdening parents/guardians. First and foremost, we select the STAI and PC-QoL because both have demonstrated reliability and repeatability and been validated. ${ }^{18} 2128$ The STAI clearly differentiates between a temporary condition and general long-standing quality of anxiety. It could help distinguish feelings of anxiety 
at a particular time from anxious personality, so we can precisely compare the outcome between time points that the parents meet the doctor with or without spirometry. The PC-QoL is used given that cough is very common symptom of children with respiratory illness and coughspecific QoL inventories for adults have been shown better specificity and sensitivity over generic QoL inventories. ${ }^{2930}$ The domains of psychological, social and physical concerns in the PC-QoL could provide insight into impacts of the intervention across aspects of life. Finally, both the questionnaires are scale-based inventories. The inventory simplicity also makes it ideal for all individuals regardless of educational backgrounds.

Another secondary outcome is doctors' and patients' opinions towards spirometry via three questions for the doctor and one question for the patient. A unipolar 10-point Likert scale model is used to measure these opinions. The scale is feasible for collecting the additional outcome since it is easy to employ and communicate. A Likert scale survey can achieve valuable data which gives insight into the complex views of participants on a single subject matter. ${ }^{17}$ Because perception of an opinion generally ranges along a continuum of positive to negative, a more refined scale with more points presumably permit individuals to express their opinions precisely and comfortably. Consequently, distortion in data decreases as the number of scale points increases, although the improvement is relatively modest beyond 5-7 points, ${ }^{31}$ hence the 10-point scale is used.

We chose to evaluate the outcomes at a single visit because spirometry information is a measure used for a single point in time for clinical decisions. However, this time frame limits us from assessing other health-related outcomes that require long-term observation such as improvement of symptoms, limitation of activities and unscheduled visits.

In summary, this RCT addresses a current gap in evidence to assess the benefit of spirometry in routine clinical practice. If this study shows that spirometry has a positive impact on clinical decision making and/or PROMs, this evidence will promote the use of spirometry as an important clinical assessment tool in multilevel care settings, especially in primary care and outreach settings where respiratory specialists are limited. Thus, respiratory healthcare for children could be optimised to maximal benefit.

Acknowledgements We are grateful to members of Cough, Airway and Asthma Research Group at Australian Centre for Health Services Innovation, particularly Anne Cook and Dan Arnold, for assisting in recruitment and database development. We also thank nurses and respiratory scientists at the respiratory clinic for supporting this study.

Contributors AC originally conceived and designed the study. IBM, WB, JM and MM co-designed the study. All authors were involved in protocol preparation. WB obtained ethics and governance, prepared study materials, recruited participants, collected data and developed database. AC and SY supervised the plan for statistical analyses and reporting. The first draft of manuscript was written by WB. All authors read and approved the final manuscript.
Funding WB is supported by a Charles Darwin University PhD scholarship. AC is supported by an Australian National Health and Medical Research Council (NHMRC) practitioner fellowship (APP1154302) and a Queensland Children's Hospital Foundation top-up grant. JM is supported by an early career fellowship grant from the Queensland Children's Hospital Foundation (RPC0772019).

Competing interests None declared.

Patient and public involvement Patients and/or the public were not involved in the design, or conduct, or reporting, or dissemination plans of this research.

Patient consent for publication Not applicable.

Provenance and peer review Not commissioned; externally peer reviewed.

Open access This is an open access article distributed in accordance with the Creative Commons Attribution Non Commercial (CC BY-NC 4.0) license, which permits others to distribute, remix, adapt, build upon this work non-commercially, and license their derivative works on different terms, provided the original work is properly cited, appropriate credit is given, any changes made indicated, and the use is non-commercial. See: http://creativecommons.org/licenses/by-nc/4.0/.

\section{ORCID iDs}

Wicharn Boonjindasup http://orcid.org/0000-0003-2942-9380

Julie M Marchant http://orcid.org/0000-0002-8614-1829

\section{REFERENCES}

1 Graham BL, Steenbruggen I, Miller MR, et al. Standardization of spirometry 2019 update. An official American thoracic Society and European respiratory Society technical statement. Am J Respir Crit Care Med 2019;200:e70-88.

2 Paton JY. A practical approach to the interpretation of lung function testing in children. Paediatr Respir Rev 2000;1:241-8.

3 Davis S. Spirometry. Paediatr Respir Rev 2006;7:S11-13.

4 Chang AB, Glomb WB. Guidelines for evaluating chronic cough in pediatrics: ACCP evidence-based clinical practice guidelines. Chest 2006;129:260S-83.

5 Debley J, Filbrun AG, Subbarao P. Clinical applications of pediatric pulmonary function testing: lung function in recurrent wheezing and asthma. Pediatr Allergy Immunol Pulmonol 2011;24:69-76.

6 Rosenfeld M, Allen J, Arets BHGM, et al. An official American thoracic Society workshop report: optimal lung function tests for monitoring cystic fibrosis, bronchopulmonary dysplasia, and recurrent wheezing in children less than 6 years of age. Ann Am Thorac Soc 2013;10:S1-11.

7 Global Initiative for Asthma. Global strategy for asthma management and prevention, 2019. Available: http://www.ginaasthma.org

8 Jat KR. Spirometry in children. Prim Care Respir J 2013;22:221-9.

9 Aurora P, Stocks J, Oliver C, et al. Quality control for spirometry in preschool children with and without lung disease. Am J Respir Crit Care Med 2004;169:1152-9.

10 Gaffin JM, Shotola NL, Martin TR, et al. Clinically useful spirometry in preschool-aged children: evaluation of the 2007 American thoracic Society guidelines. J Asthma 2010;47:762-7.

11 Nair SJ, Daigle KL, DeCuir P, et al. The influence of pulmonary function testing on the management of asthma in children. $J$ Pediatr 2005;147:797-801.

12 Holt EW, Tan J, Hosgood HD. The impact of spirometry on pediatric asthma diagnosis and treatment. J Asthma 2006;43:489-93.

13 Abramson MJ, Schattner RL, Holton C, et al. Spirometry and regular follow-up do not improve quality of life in children or adolescents with asthma: cluster randomized controlled trials. Pediatr Pulmonol 2015;50:947-54.

14 Dombkowski KJ, Hassan F, Wasilevich EA, et al. Spirometry use among pediatric primary care physicians. Pediatrics 2010;126:682-7.

15 Blain EA, Craig TJ. The use of spirometry in a primary care setting. Int J Gen Med 2009;2:183-6.

16 Bianchi M, Clavenna A, Bonati M. Spirometry remains an unfulfilled right for children with asthma. J Pediatr 2015;166:1325-6.

17 Likert R. A technique for the measurement of attitudes. Archives of Psychology 1932;22:55.

18 Spielberger C, Gorsuch R, Lushene R, et al. Manual for the StateTrait anxiety inventory (form Y1 - Y2), 1983.

19 Spielberger CD. State-trait anxiety inventory: a comprehensive bibliography. Consulting Psychologists Press, 1989.

20 Newcombe PA, Sheffield JK, Juniper EF, et al. Development of a parent-proxy quality-of-life chronic cough-specific questionnaire: clinical impact vs psychometric evaluations. Chest 2008;133:386-95. 
21 Newcombe PA, Sheffield JK, Juniper EF, et al. Validation of a parentproxy quality of life questionnaire for paediatric chronic cough (PCQOL). Thorax 2010;65:819-23.

22 Chan A-W, Tetzlaff JM, Gøtzsche PC, et al. Spirit 2013 explanation and elaboration: guidance for protocols of clinical trials. $B M J$ 2013;346:e7586.

23 Beydon N, Davis SD, Lombardi E, et al. An official American thoracic Society/European respiratory Society statement: pulmonary function testing in preschool children. Am J Respir Crit Care Med 2007; 175:1304-45.

24 Quanjer PH, Stanojevic S, Cole TJ, et al. Multi-Ethnic reference values for spirometry for the 3-95-yr age range: the global lung function 2012 equations. Eur Respir J 2012;40:1324-43.

25 Newcombe PA, Sheffield JK, Chang AB. Minimally important change in a Parent-Proxy quality-of-life questionnaire for pediatric chronic cough. Chest 2011;139:576-80.

26 Goyal V, Grimwood K, Ware RS, et al. Efficacy of oral amoxicillinclavulanate or azithromycin for non-severe respiratory exacerbations in children with bronchiectasis (BEST-1): a multicentre, three-arm, double-blind, randomised placebo-controlled trial. Lancet Respir Med 2019;7:791-801.

27 Goyal V, Grimwood K, Byrnes CA, et al. Amoxicillin-Clavulanate versus azithromycin for respiratory exacerbations in children with bronchiectasis (BEST-2): a multicentre, double-blind, non-inferiority, randomised controlled trial. Lancet 2018;392:1197-206.

28 Guillén-Riquelme A, Buela-Casal G. [Meta-analysis of group comparison and meta-analysis of reliability generalization of the State-Trait Anxiety Inventory Questionnaire (STAI)]. Rev Esp Salud Publica 2014;88:101-12.

29 French CT, Irwin RS, Fletcher KE, et al. Evaluation of a coughspecific quality-of-life questionnaire. Chest 2002;121:1123-31.

30 Birring SS, Prudon B, Carr AJ, et al. Development of a symptom specific health status measure for patients with chronic cough: Leicester cough questionnaire (LCQ). Thorax 2003;58:339-43.

31 Krosnick JA, Fabrigar LR. Designing rating scales for effective measurement in surveys. In: Survey measurement and process quality, 1997: 141-64. 